\title{
On methodology of measuring parameters with the increased sensitivity to residual or irradiation induced inhomogeneities in semiconductors
}

\author{
G.P. Gaidar \\ Institute for Nuclear Researches, NAS of Ukraine \\ 47, prospect Nauky, 03680 Kyiv, Ukraine; e-mail: gaidar@kinr.kiev.ua
}

\begin{abstract}
Within the frame of theory of anisotropic scattering, it was studied the relation of values for specific resistance changes under the axial elastic deformations for manyvalley semiconductors, $n$-Ge and $n$-Si. The aspect ratio between values of the saturated

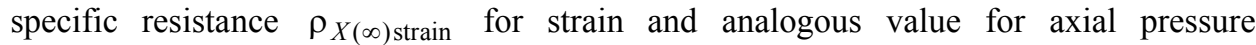

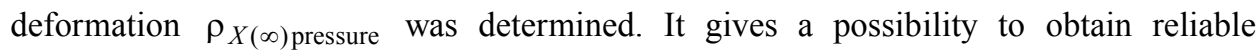
information concerning the value $\rho_{X(\infty) \text { strain }}$ even for the case when mobility of carriers and, consequently, the value $\rho=\rho(X)$ are significantly decreased, for example, under irradiation treatment of crystals.
\end{abstract}

Keywords: many-valley semiconductors, piezoresistance, mechanical stress, axial pressure, strain, parameter of scattering anisotropy.

Manuscript received 30.04.09; accepted for publication 10.09.09; published online 30.10.09.

\section{Introduction}

During experimental study of properties of semiconductors, researchers often face up with the problem to determine low value or to characterize parameters that are very sensitive to insignificant heterogeneities present in crystal. However, in the case when hard determined parameters can be expressed through parameters determined simpler, one can perform experimental measurements of the latter ones and then calculate the former by using the established relations.

Namely, this technique was used in the monograph [1] to determine the value of magnetoresistance $\rho_{0}^{H(\infty)}$ for the classically strong magnetic field $H(\infty)$, taking into account the hardness of its measurements due to the significant sensitivity of magnetoresistance to the residual crystal heterogeneity in the region of strong magnetic fields.

It was found that piezoresistance $\rho_{X(\infty)}^{0}$ in the region of saturation $X(\infty)$ had low sensitivity to the residual heterogeneity of specific resistance. So, using the only value $\rho_{X(\infty)}^{0}$ measured in absence of magnetic field, the authors proposed to calculate a desired value of the magnetoresistance $\rho_{0}^{H(\infty)}$ by the relation:

$$
\rho_{0}^{H(\infty)}=\frac{K+8}{7 K+2} \rho_{X(\infty)}^{0},
$$

where $H$ is the magnetic field; $X$ is the mechanical stress $\left(\mathrm{kgf} / \mathrm{cm}^{2}\right) ; \quad K=\frac{\mu_{\perp}}{\mu_{\|}}$is the parameter of mobility anisotropy; $\mu_{\|}, \mu_{\perp}$ are the mobilities of carriers along and crosswise of the main axis of isoenergetic ellipsoid. The value of the parameter of mobility anisotropy $K$ in the single isoenergetic ellipsoid is determined from above mentioned measurements of piezoresistance in the range of saturation by the simple relation: $K=\frac{3}{2} \frac{\rho_{X(\infty)}^{0}}{\rho_{0}}-\frac{1}{2}$, where $\rho_{0}$ is the specific resistance of unstressed crystal. Parameter $K$ is the certain number at the $N \equiv n_{e}=$ const, where $n_{e}$ is the total concentration of current carriers in crystal, which coincides with the concentration of impurity dopants and doesn't depend on $X$.

\section{The technique of determination of strain piezoresistance}

Let us consider, for example, the germanium monocrystal of $n$-type conductivity $(n-\mathrm{Ge})$. Under the 
current passing with the density $j$ and in the presence of mechanical pressure $X$ along the crystallographic axis $\langle 111\rangle$, i. e. along the main axis of one of four equivalent isoenergetic ellipsoids, the noted ellipsoid will go down and three others will go up along the energy scale. It causes distribution of current carriers between the energy minimums in the conduction band. As a result, under the comparatively high stress $X \|\langle 111\rangle$ all electrons can pass in single ellipsoid oriented in the direction of the deformation axis with a minimal value of current carrier mobility $\mu=\mu_{\|}$. Due to this distribution, the specific resistance of crystal, which is measured in the same direction $(j \|\langle 111\rangle)$, is significantly increased with the increasing pressure of mechanical stresses $X$ up to saturation, which is described by the expression $\rho_{X(\infty)}^{\langle 111\rangle}=\frac{1}{e n_{e} \mu_{\|}}=$const, where $\rho_{X(\infty)}^{\langle 111\rangle}$ is the piezoresistance in the region of saturation; $e$ is the electron charge; $n_{e}=4 N_{0}$ is the total concentration of electrons; $N_{0}$ is the concentration of electrons in each ellipsoid in absence of crystal deformation, $\mu_{\|}$is the mobility of electrons along the main axis of isoenergetic ellipsoid, which corresponds to the maximal effective mass $m_{\|}$. In this case, the parameter of mobility anisotropy can be expressed as

$K=\frac{3}{2} \frac{\rho_{X(\infty) \text { pressure }}^{\langle 111\rangle}}{\rho_{0}}-\frac{1}{2}$.

It is reasonable to put the question, do we have to use well measured values of pressure piezoresistances to determine small values of piezoresistances of strain (along crystallographic direction $\langle 111\rangle$ for the particular case of $n-\mathrm{Ge})$ ?

Under the investigation of concentration dependence of the parameter of mobility anisotropy $K=\frac{\mu_{\perp}}{\mu_{\|}}=K\left(n_{e}\right)$, as it was found in [2], despite the simplicity of the connection $K$ with $\mu_{\perp}$ and $\mu_{\|}$, only $\mu_{\perp}$ depends on $n_{e}$. Thus, even at presence of useful connection between $\rho_{X(\infty) \text { strain }}$ and $\rho_{X(\infty) \text { pressure }}$ it was necessary to make sure that at both limits of concentration ranges (i. e. at $n_{e}{ }^{\min }$ and $n_{e}{ }^{\max }$ ) the condition $\rho_{X(\infty) \text { strain }}<\rho_{X(\infty) \text { pressure }}$ is remained valid.

To realize the proposed technique let us use equation, which was determined in [3] for the pressure piezoresistance

$\frac{\rho_{X(\infty) \text { pressure }}^{\langle 111\rangle}}{\rho_{0}}=\frac{1}{4}\left(\frac{8 K+1}{3}+1\right)$

and the strain piezoresistance

$$
\frac{\rho_{X(\infty) \text { strain }}^{\langle 111\rangle}}{\rho_{0}}=\frac{3}{4}\left(\frac{3}{8 K+1}+1\right) \text {. }
$$

After elimination from the system of equations (3) and (4) the value of $\rho_{0}$, after simple algebraic transforms it is obtained:

$\rho_{X(\infty) \text { strain }}^{\langle 111\rangle}=9 \frac{\rho_{X(\infty) \text { pressure }}^{\langle 111\rangle}}{8 K+1}$,

and the parameter of mobility anisotropy for single isoenergetic ellipsoid $K=\mu_{\perp} / \mu_{\|}$can be obtained from the same measurement of pressure piezoresistance $\rho_{X(\infty) \text { pressure }}$ using the relation (2).

It is known that in $n$-Ge when the concentration of current carries varies by five orders (from $10^{16}$ up to $10^{12} \mathrm{~cm}^{-3}$ ) the parameter of mobility anisotropy $K$ (at $77 \mathrm{~K}$ ) increases only from 7.4 up to 16 . Using the equation (5) valid in the case of non-degenerate electron gas, let us estimate by how many orders the $\alpha$-value will exceed unity at the concentration limits (at which, even at $70 \mathrm{~K}$, the electron gas remains non-degenerated), i. e.:

$\alpha \equiv \frac{\rho_{X(\infty) \text { pressure }}^{\langle 111\rangle}}{\rho_{X(\infty) \text { strain }}^{\langle 111\rangle}}>1$.

It was found that in relatively pure (without impurities) crystal of $n$-Ge with the concentration $n_{e} \equiv N=10^{12} \mathrm{~cm}^{-3}$ the $\alpha$-value is 14.33 . But even in comparatively strongly doped $n$-Ge with $n_{e} \equiv N=10^{16} \mathrm{~cm}^{-3}$, the $\alpha$-value is still 6.69. This means that the appropriateness of proposed technique of the determination of value for the strain piezoresistances from the measurements of the pressure piezoresistances is justified for all the considered range of concentrations, which covers five orders.

In the case of $n-\mathrm{Ge}$, for the equation (5) to be used at any given concentration $n_{e}$ (within the considered concentration range), it is possible to use the data of the dependence $K=K\left(n_{e}\right)$ presented in the monograph ([1], p. 95), and for $n$-Si such dependence is shown in Fig. $1[4]$.

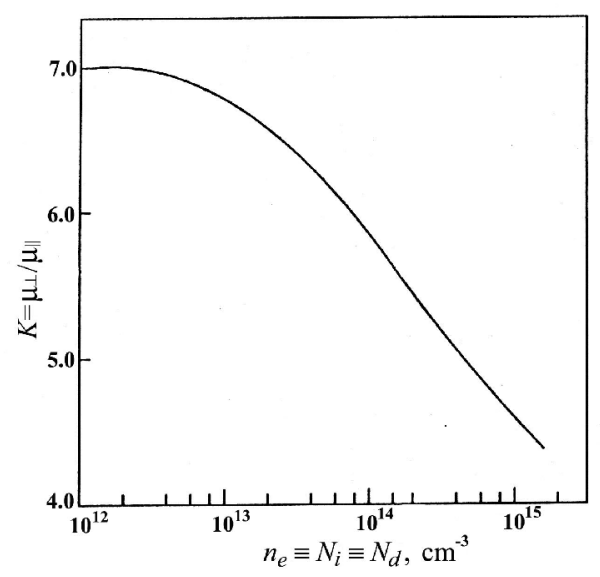

Fig. 1. Dependence of the parameter of mobility anisotropy $K=\mu_{\perp} / \mu_{\|}$on the concentration of ionized impurities $N_{i} \equiv n_{e}$ in the region of mixed scattering at $T=77.4 \mathrm{~K}=$ const for the crystals $n$-Si. Dependence was obtained by the calculations in the frame of the theory of anisotropic scattering. 
In general, the value $K$ (for an arbitrary concentration at which the degeneration of electron gas is not observed at the temperature of piezoresistances measurements $\sim 77 \mathrm{~K}$ ) can be also evaluated (both for $n$ Ge and $n$-Si) from the theory of anisotropic scattering [5] by following equation:

$$
K=\frac{\mu_{\perp}}{\mu_{\|}}=\frac{m_{\|}}{m_{\perp}} \frac{a_{\perp}}{a_{\|}} \frac{I_{2}}{I_{1}},
$$

where $m_{\|}$and $m_{\perp}$ are the cyclotron effective masses for single isoenergetic ellipsoid along the main axes and crosswise for them, respectively.

$$
\left.\begin{array}{l}
\left.\begin{array}{l}
m_{\|}=1.580 m_{0} \\
m_{\perp}=0.082 m_{0}
\end{array}\right\} \quad \text { for } n \text {-Ge and } \\
m_{\|}=0.910 m_{0} \\
m_{\perp}=0.191 m_{0}
\end{array}\right\} \quad \begin{aligned}
& \text { for } n-\mathrm{Si} \\
&
\end{aligned}
$$

$m_{0}$ is the mass of free electron.

$a_{\|}=3.40 \cdot 10^{-17} \mathrm{erg}^{1 / 2}{ }^{\circ} \mathrm{C} \cdot \mathrm{s}$

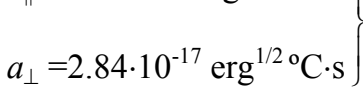
for $n-\mathrm{Ge}$ and

$a_{\|}=3.09 \cdot 10^{-17} \mathrm{erg}^{1 / 2}{ }^{\mathrm{o}} \mathrm{C} \cdot \mathrm{s}$

$\left.a_{\perp}=4.67 \cdot 10^{-17} \mathrm{erg}^{1 / 2} \mathrm{o} \cdot \mathrm{s}\right\} \quad$ for $n-\mathrm{Si}$

The equations for calculation of integrals $I_{1}$ and $I_{2}$ for $n$-Ge and $n$-Si are presented in Appendix.

Note, that under the using of equations (2) and (5) in the case of $n$-Si it should be taken into account the orientation of isoenergetic ellipsoids by the change of $\rho_{X(\infty) \text { pressure }}^{\langle 111\rangle}$ and $\rho_{X(\infty) \text { strain }}^{\langle 111\rangle}$ on $\rho_{X(\infty) \text { pressure }}^{\langle 100\rangle}$ and $\rho_{X(\infty) \text { strain }}^{\langle 100\rangle}$. It reflects the fact that strain piezoresistances in $n$ - $\mathrm{Si}$, in contrast to $n$-Ge, must be measured at the condition $X\|j\|\langle 100\rangle$, i.e. the mechanical stress $X$ and electric current $j$ have direct along the crystallographic orientation $\langle 100\rangle$ of the sample.

\section{Conclusions}

In conclusion to above mentioned it should be claimed that:

1) there is a simple relation between the change of the specific resistance in many-valley semiconductor of $n$-Ge type under axial deformation of pressure and the change of specific resistance, which arise under the axial deformation of strain;

2) both for $n$-Ge, which is free of dopants, and for the crystals with a relatively high doping level, which, however, don't lead to the degeneration of electron gas at temperature of piezoresistances measurements (near
$77 \mathrm{~K}$ ), the value of pressure piezoresistances is practically higher by one order in comparison with strain piezoresistances, and consequently, the proposed in p. 1 technique of evaluation of strain piezoresistances by using the experimental data for pressure piezoresistances for all above mentioned concentration range can be considered as acceptable and physically reasonable;

3) approaches and conclusions proposed in this paper can be applied not only to $n$-Ge and $n$-Si but also to another many-valley semiconductors after the same technical modification.

The described approach to methodology of determination of some parameters inherent to semiconductors with low values or high sensitivity to residual (or induced by radiation treatment $[6,7]$ ) crystal inhomogeneities, which is hard eliminated in principle, can be very useful under the assumption that above mentioned limitations are fulfilled.

\section{Appendix}

The general view of the integrals $I_{1}$ and $I_{2}$ for $n$-Ge and $n-\mathrm{Si}$ :

$$
\begin{aligned}
& I_{1}=\int_{0}^{\infty} \frac{e^{-x} x^{3} d x}{x^{2}+b_{0}} ; I_{2}=\int_{0}^{\infty} \frac{e^{-x} x^{3} d x}{x^{2}+b_{1}} ; \\
& 2.65 \cdot 10^{5} \frac{a_{\|} N}{T^{3}}\left(32.0+\ln \frac{T^{2} x}{n^{\prime}}+1.26 \cdot 10^{-14} \frac{n^{\prime}}{T^{2} x}\right) \\
& \quad \text { in case of } n-\mathrm{Ge} \\
& 9.68 \cdot 10^{5} \frac{a_{\|} N}{T^{3}}\left(32.0+\ln \frac{T^{2} x}{n^{\prime}}+10^{-14} \frac{n^{\prime}}{T^{2} x}\right) \\
& \text { in case of } n-\mathrm{Si}
\end{aligned} ;
$$$$
b_{1}=\left\{\begin{array}{r}
3.23 \cdot 10^{6} \frac{a_{\perp} N}{T^{3}}\left(31.0+\ln \frac{T^{2} x}{n^{\prime}}+2.8 \cdot 10^{-14} \frac{n^{\prime}}{T^{2} x}\right) \\
\text { in case of } \begin{array}{r}
n-\mathrm{Ge} \\
3.47 \cdot 10^{6} \frac{a_{\perp} N}{T^{3}}\left(31.4+\ln \frac{T^{2} x}{n^{\prime}}+1.46 \cdot 10^{-14} \frac{n^{\prime}}{T^{2} x}\right) \\
\text { in case of } n-\mathrm{Si}
\end{array} ;
\end{array}\right.
$$

$N=N_{d}+N_{a}$ is the total concentration of impurities in crystal; $n^{\prime}=N_{d}-N_{a}$ (in the condition of lack of compensating dopants $N_{a}=0$ and $n^{\prime}=n_{e}=N$ ). It is clear that $b_{0}$ and $b_{1}$ are dependent on temperature, total concentration of impurities in crystal, the degree of compensation, and are different for $n-\mathrm{Ge}$ and $n-\mathrm{Si}$.

\section{References}

1. P.I. Baranskii, I.S. Buda, I.V. Dakhovskii, V.V. Kolomoets, Electrical and Galvanomagnetic Phenomena in Anisotropic Semiconductors. Naukova Dumka, Kiev, 1977 (in Russian). 
2. P.I. Baranskii, E.F. Venger, G.P. Gaidar, Determination of the reasons of decreasing of anisotropy parameter $K=\mu_{\perp} / \mu_{\|}$in $n$-Ge under the increasing of impurity scattering contribution // Dopovidi NAN Ukrainy № 9, p. 84-86, 1999 (in Ukrainian).

3. P.I. Baranskii, I.S. Buda, I.V. Dakhovskii, V.V. Kolomoets, A.G. Samoilovich, Piezoresistance of $n$-Ge in $\langle 111\rangle$ direction under the condition of mixed scattering // Fizika tekhnika poluprovodnikov 8, N. 5, p. 984-986 (1974) (in Russian).

4. G. P. Gaidar, The features of the scattering anisotropy in the n-Si and n-Ge crystals which appear under the influence of the axial elastic deformation, heat treatment and the nuclear irradiation. Thesis for candidate's degree in Physics and Mathematics in the speciality 01.04.07 "Solid State Physics", Kyiv, 2001 (in Ukrainian).

5. A.G. Samoilovich, I.S. Buda, I.V. Dakhovskii, Theory of anisotropic scattering // Fizika tekhnika poluprovodnikov 7, N. 4, p. 859 (1973) (in Russian).

6. I.D. Konozenko, A.K. Semenuk, V.I. Khivrich, Radiation Effects in Silicon. Naukova Dumka, Kiev, 1974 (in Russian).

7. A.K. Semenuk, Radiation Effects in Many-Valley Semiconductors. Nadstyr'e Publ., Lutsk, 2001 (in Ukrainian). 